\title{
Malignant gastroin- testinal stromal cell tumour (GIST) of the stomach
}

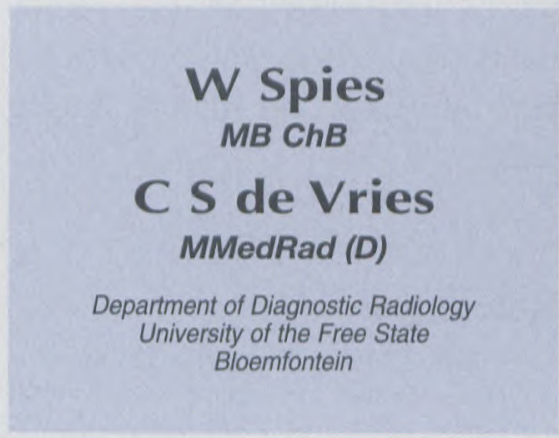

\section{Case presentation}

A 55-year-old female patient presented with a painful mass in the left hypochondrium.

Sonar revealed a well-demarcated round thin-walled lesion with internal echoes with a homogeneous pattern (Fig. 1). The mass measured approximately 15 x $13 \mathrm{~cm}$. Duplex Doppler revealed a low venous flow pattern, and a sonar-guided fine needle aspiration (FNA), revealed a bloody aspirate (Fig. 2). Computerised tomography (CT) was done immediately after ultrasound investigation. No oral contrast was given. The cystic lesion (Hounsfield unit $(\mathrm{HU})=18$ on precontrast study) was situated adjacent to the stomach in the lesser omental sac. It was difficult to differentiate the cystic lesion from either the stomach or the pancreas on CT. The splenic artery could also not be differentiated from the cystic lesion. The post-contrast CT showed rim enhancement,

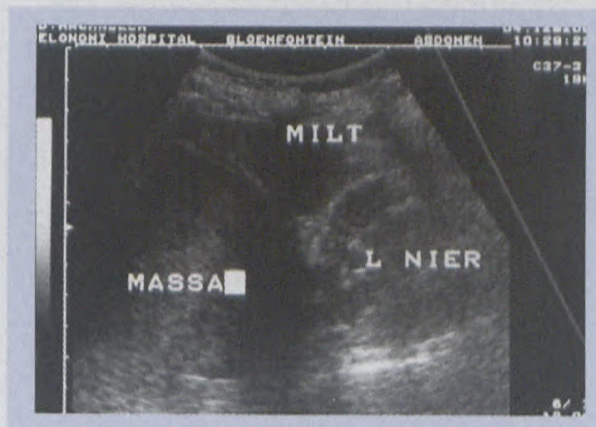

Fig. 1. Sonar of a thin-walled lesion with internal echoes.

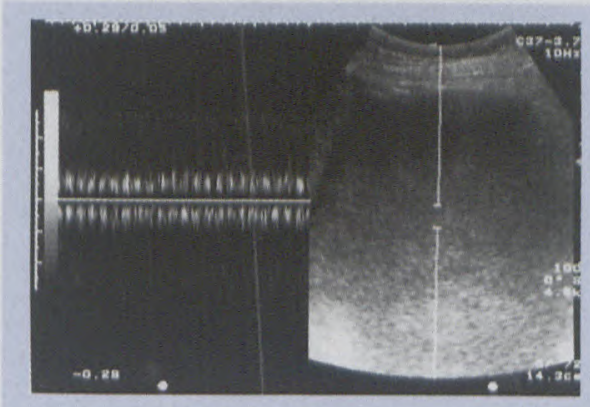

Fig. 2. Duplex Doppler revealing low venous flow pattern.

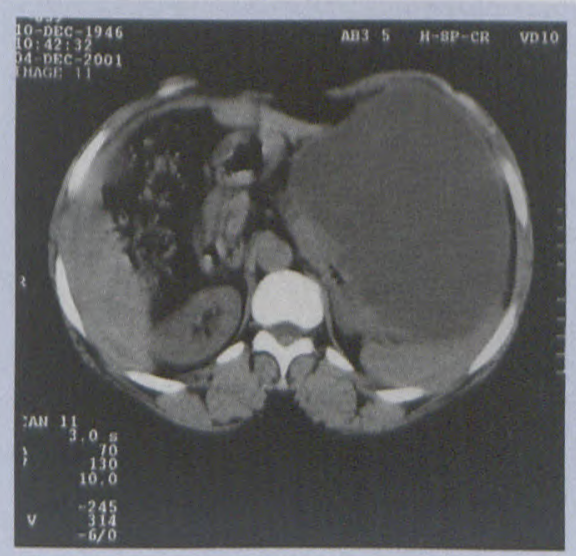

Fig. 3. Pre-contrast CT.

but no enhancement of the contents was apparent (Figs 3 and 4).

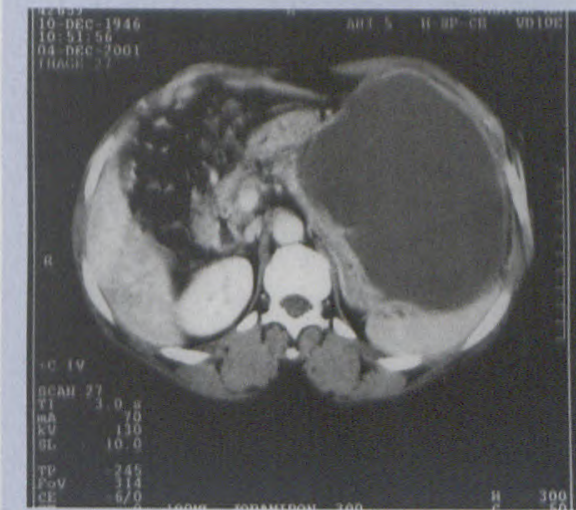

Fig. 4. Post-contrast CT.

The preliminary differential diagnosis included a haemorrhagic pseudocyst, thrombosed splenic artery aneurysm, subacute haematoma, or abscess. A splenic, hepatic and superior mesenteric artery (SMA) angiogram revealed no significant pathology, splenic artery aneurysm, or neovascularity. A surgical exploration and resection was then done. Stomach wall slices showed the presence of a cellular tumour with small areas of necrosis. Evidence of spindle-shaped and epithelioid cells was found. A slight pleomorphism was present with an average of 2 mitosis per 10 high-field magnification.

The final diagnosis was a malignant gastrointestinal stromal cell tumour (GIST).

\section{Discussion}

GISTs are uncommon mesenchymal tumours of the gastrointestinal (GI) tract. GISTs include tumours previously designated as leiomyoma, cellular leiomyoma, leiomyoblastoma and leiomyosarcoma. The oesophagus is the exception, with leiomyoma the most common mesenchymal tumour. Studies show that phenotypically undifferentiated GISTs are also genetically different from leiomyomas and schwannomas and support their clas- 
sification apart from leiomyomas. ${ }^{1}$ GISTs are composed of spindle (70\%) or epithelioid (30\%) cells. $10-30 \%$ are malignant, showing intra-abdominal spread and/or liver metastasis.

GISTs are both malignant and benign. They may be submucosal, subserosal or intraluminal. The CT appearance of a GIST may include a sharply defined mass with homogeneous attenuation, sometimes with clarification.

A study by Kim et $a l^{2}$ analysed the clinical presentation, pathology and long-term follow-up of 19 patients with GIST tumours (12 gastric, 2 duodenal, 3 jejunal and 2 rectal). The most common clinical presentation was GI bleeding. CT, contrast studies, and endoscopy were used to identify tumour mass. In only two instances could the diagnosis of GIST be made preoperatively.
Histology was variable and the size of the tumours ranged from 0.8 to $23 \mathrm{~cm}$. The clinical course is favourable with complete resection.

Needle biopsy can be used for the diagnosis of GIST tumours preoperatively. GIST can be classified as benign, borderline, or malignant using immunochemistry, size, mitotic activity and clinical outcome. Spindle cell and epithelioid types can be distinguished. Cytomorphology alone cannot be used to assess malignancy and predictions of aggressiveness but must be interpreted with a gross and histological examination of the resected specimen. Immunocytochemical staining for $\mathrm{CD}$ is helpful in confirming the diagnosis. Care must be taken to differentiate epithelioid-type GISTs from adenocarcinoma. GISTs are also immunohistochemically positive for C-kit (CD117), CD34 and sometimes for actin, but are mostly negative for desmin and S- 100 protein. The malignant GIST especially, shows activating mutations in the C-kit gene. GIST and GI autonomic nerve tumours (GANTs) can overlap. The cell of origin is not well understood, but resemblance to the interstitial cells of Gajal, expression of the smooth muscle markers and occurrence outside of the GI tract suggest an origin from multipotential cells that can differentiate into Cajal and smooth muscle cells.

\section{References}

1. Dodd LG, Nelson RC, Mooney EE, Gottfried $\mathrm{M}$. Fine-needle aspiration of gastrointestinal stromal tumours. Am J Clin Pathol 1998; 109: 439-443.

2. Kim CJ, Day S, Yeh KA. Gastrointestinal stromal tumours: analysis of clinical and pathologic factors. Am Surg 2001; 67: 135-137. 